\title{
Canadian Emergency Medicine and Critical Care Physician Perspectives on Pandemic Triage in COVID-19
}

Ali Mulla ( $\sim$ ali.mulla@medportal.ca )

University of Toronto, Department of Medicine https://orcid.org/0000-0002-2442-1542

Blair L. Bigham

Stanford University Medical Centre

Andrea Frolic

Hamilton Health Sciences, Hamilton, Canada; McMaster University, Hamilton, Canada

Michael D. Christian

London's Air Ambulance, Barts NHS Health Trust, London, England.

\section{Short Report}

Keywords: COVID-19, Triage, Surge Capacity, Psychological Stress

Posted Date: July 29th, 2020

DOI: https://doi.org/10.21203/rs.3.rs-50315/v1

License: (a) (i) This work is licensed under a Creative Commons Attribution 4.0 International License. Read Full License 


\section{Abstract}

Purpose: Local and regional policies to guide the allocation of scarce critical care resources have been developed, but the views of prospective users are not understood. We sought to investigate the perspectives of Canadian acute care physicians towards triaging scarce critical care resources in the COVID-19 pandemic.

Methods: We rapidly deployed a brief survey to Canadian emergency and critical care physicians in April 2020 to investigate current attitudes towards triaging scarce critical care resources and identify subsequent areas for improvement. Descriptive and between-group analyses along with thematic coding were used.

Results: The survey was completed by 261 acute care physicians. Feelings of anxiety related to the pandemic were common (65\%), as well as fears of psychological distress if required to triage scarce resources $(77 \%)$. Only $49 \%$ of respondents felt confident in making resource allocation decisions. Both critical care and emergency physicians favored multidisciplinary teams over single physicians to allocate scarce critical care resources. Critical care physicians were supportive of decision making by teams not involved in patient care ( $3.4 / 5$ vs $2.9 / 5 p=0.04)$, whereas emergency physicians preferred to maintain their involvement in such decisions ( $3.4 / 5$ vs $4.0 / 5 p=0.007)$. Free text responses identified five themes for subsequent action including the need for further guidance on existing triage policies, ethical support in decision making, medico-legal protection, additional tools for therapeutic communications, and healthcare provider psychological support.

Conclusion: There is an urgent need for collaboration between policymakers and frontline physicians to develop critical care resource triage policies that wholly consider the diversity of provider perspectives across practice environments.

\section{Introduction}

Following the identification of a novel coronavirus (SARS-CoV-2) and Coronavirus Disease 19 (COVID19), Canadian governments and hospitals have re-evaluated pandemic response protocols to address issues related to infection control, surge capacity, and healthcare provider (HCP) psychological safety. In response to the overwhelming demands for critical care services including limited ventilators and critical care beds in China, Italy, and New York State, new regional protocols to triage which patients are allocated such resources have been developed. ${ }^{1-3}$

Physicians engaged in the rationing of scarce critical care resources are expected to be at an increased risk of moral and psychological distress. ${ }^{4,5}$ Negative psychological effects seen in SARS have raised concerns about similar outcomes in COVID-19 including anticipatory guilt, physician burnout, anxiety, depression, and moral injury. ${ }^{5}$ We aimed to understand the attitudes of critical care and emergency medicine physicians towards critical care resource triage and moral injury in the COVID-19 pandemic. 


\section{Methods}

This study was approved by the Hamilton Integrated Research Ethics Board (\#10844) and informed consent was obtained. We conducted a voluntary English electronic survey of Canadian emergency and critical care physicians. No incentives were offered. Development involved three design and feedback cycles and pilot testing. Fifteen questions presented over eight screens with response formats including 5-point likert scale, multiple-choice, and free text were asked. Adaptive questions and completeness checks were used where appropriate.

We opted for a convenience sample of physician members of the Canadian Critical Care Society and Canadian Association of Emergency Physicians by email invitation. Convenience sampling was preferred to probabilistic sampling due to the urgency of examining physician attitudes towards established triage guidelines and the difficult-to-reach target population, which was engaged heavily in responding to the pandemic. ${ }^{6}$ Further, the homogenous target population we sampled would offer more generalizability than conventional convenience sampling, and probability sampling would have led to delays in answering these urgent questions. ${ }^{7}$ An invitation to complete a short electronic questionnaire was sent weekly for three weeks. All data was collected anonymously using REDCap (Research Electronic Data Capture), a secure, web-based research software platform hosted by the University. ${ }^{8,9}$

Incomplete surveys were included in the analysis. Likert-scale responses were analysed as continuous data; other data was treated categorically. A preplanned analysis of between group comparisons of residents versus staff physicians and emergency medicine versus critical care physicians was done by submitting means and proportions to a Student's T-test or Chi Square/Fisher's Exact test, respectively. We used Stata/SE 13.1 (StatCorp, College Station, TX, USA). A two-tailed $p<0.05$ was considered significant. Qualitative data was analysed via an inductive thematic analysis, with themes coded independently by two investigators then agreed upon by consensus (BB, AM).

\section{Results}

There were 261 physician responses with a completion rate of $84 \%$. Respondents worked in Canada (98.2\%), at hospitals with greater than 400 beds $(71.7 \%)$, in emergency medicine $(68.9 \%)$, and critical care (32.9\%), treating adults (94.5\%), and children (44.3\%). ${ }^{10}$ Residents and clinical fellows constituted $23.3 \%$ of all responses, with the remainder being staff physicians. Respondents were evenly distributed across seniority categories $(0-5$ years $27.9 \%, 6-10$ years $25.6 \%, 10-15$ years $15.6 \%$, more than 15 years $31.1 \%$ ).

Most respondents (86.1\%) felt confident or very confident in performing their clinical role in the pandemic. Only $48.7 \%$ felt confident or very confident in their ability to decide who should get a ventilator if resources were scarce. Factors associated with greater confidence in triaging ventilators included knowledge (91.2\%), hospital policies (48.7\%), existing evidence $(77.0 \%)$, and consultation with other physicians (76.1\%). Factors associated with a lack of confidence included lack of knowledge (50.0\%), 
lack of previous experience triaging critical care resources (71.2\%), medicolegal liability concerns (57.7\%), ethical (75.0\%), and personal moral concerns (69.2\%).

Between group comparisons found that residents were less confident than attendings in their role $(p<0.001)$ and ability to triage critical care resources $(p<0.001)$. Critical care physicians were more confident than emergency physicians in their role $(4.5 / 5 \mathrm{vs} 4.1 / 5 \mathrm{p}=0.001)$ and ability to triage scarce resources $(3.7 / 5$ vs $3.2 / 5, p=0.001)$.

Both critical care and emergency physicians favored multidisciplinary teams over single physicians to allocate scarce critical care resources in crisis scenarios but differed with respect to most responsible physician (MRP) inclusion (Table 1). Critical care physicians were more likely to favor decision making by teams not involved in patient care $(3.4 / 5$ vs $2.9 / 5 p=0.04)$ compared to emergency physicians who preferred to maintain MRP involvement $(3.4 / 5$ vs $4.0 / 5 p=0.007)$.

Table 1 Critical care resource allocation decision making $(n=221)$

\begin{tabular}{|c|c|c|c|c|c|}
\hline & \multicolumn{5}{|c|}{ Number (\%) } \\
\hline & $\begin{array}{l}\text { Strongly } \\
\text { Disagree } \\
\text { (1) }\end{array}$ & $\begin{array}{l}\text { Somewhat } \\
\text { Disagree } \\
\text { (2) }\end{array}$ & $\begin{array}{l}\text { Neutral } \\
\text { (3) }\end{array}$ & $\begin{array}{l}\text { Somewhat } \\
\text { Agree } \\
\text { (4) }\end{array}$ & $\begin{array}{l}\text { Strongly } \\
\text { Agree } \\
\text { (5) }\end{array}$ \\
\hline MRP & $46(20.8)$ & 45 (20.4) & $\begin{array}{l}44 \\
(19.9)\end{array}$ & $61(27.6)$ & $\begin{array}{l}25 \\
(11.3)\end{array}$ \\
\hline $\begin{array}{l}\text { Multidisciplinary team, } \\
\text { including MRP }\end{array}$ & $12(5.4)$ & $21(9.5)$ & $\begin{array}{l}30 \\
(13.6)\end{array}$ & 77 (34.8) & $\begin{array}{l}81 \\
(36.7)\end{array}$ \\
\hline $\begin{array}{l}\text { Multidisciplinary team, not } \\
\text { involved in patient care }\end{array}$ & 47 (21.3) & $45(20.4)$ & $\begin{array}{l}27 \\
(12.2)\end{array}$ & $45(20.4)$ & $\begin{array}{l}57 \\
(25.8)\end{array}$ \\
\hline $\begin{array}{l}\text { Single triage physician not } \\
\text { involved in patient care }\end{array}$ & $94(42.5)$ & $56(25.3)$ & $\begin{array}{l}34 \\
(15.4)\end{array}$ & $26(11.8)$ & $11(5.0)$ \\
\hline
\end{tabular}

MRP = most responsible physician (involved in patient care)

Most respondents reported feeling anxious due to the pandemic (6.4\% not at all anxious, $21.0 \%$ somewhat anxious, $28.3 \%$ neutral, $39.3 \%$ moderately anxious, $5.0 \%$ very anxious). Many physicians had concerns about the negative psychological impact that engaging in critical care resource allocation may result in $(7.4 \%$ not at all concerned, $19.7 \%$ somewhat concerned, $15.7 \%$ neutral, $35.8 \%$ moderately concerned, $21.4 \%$ very concerned).

Respondents reported high levels of confidence in the surge-capacity of their health system to meet demands for critical care resources in COVID-19, with $13.7 \%$ not at all or somewhat confident, and $67.6 \%$ confident or very confident. Additionally, COVID-19 was found to have bolstered Canadian physician commitment to their medical careers with $40.2 \%$ more committed and $9.6 \%$ less committed. 
Five themes were identified by consensus from responses to the free text question: "What resources would be helpful to reduce moral distress and the negative psychological impact of triaging critical care resources?". Themes included the need for guidance on existing triage policies, bioethicist support in decision making, health care provider medico-legal protection, therapeutic communications support, and peer-support and physician psychological services.

First, respondents requested a clear policy or guideline endorsed by a professional association or government. They requested that guidelines be evidence based, and easy to use, noting that existing guidelines are too vague and complex to be used in a crisis setting. A need for consistency and review of provincial and regional protocols, along with educational outreach to disseminate established protocols was also noted.

Second, respondents were aligned in their desire to have teams, rather than individuals, make triage decisions. Many desired a bioethicist to be part of these teams. While most physicians wanted third-party teams to make triage decision, some expressed a desire to be a part of the decision making process for the patients they were caring for.

Third, respondents expressed concern that their involvement in triage decisions may result in civil litigation or regulatory body discipline. They requested the support of their hospitals, professional colleges, and regulatory bodies along with the "legislative protection of health care providers involved in making such decisions".

Fourth, respondents felt ill-equipped to have conversations with patients and families about resource triage decisions. Many worried that there would be no time to communicate with patients and families if there was an overwhelming surge in patients. Supports such as scripts, "written materials to give to patients and families", allied health professionals including social workers were desired.

Lastly, respondents were worried for their own mental health if faced with an overwhelming demand for critical care resources, with many referencing the events in China, Italy and New York as current stressors. Clinicians acknowledged the great psychological burden placed on them if forced to ration critical care resources, with one describing it as "unprecedented and antithetical to Canadian healthcare".Suggested interventions to support mental wellbeing, including peer support teams, professional counselling and formal debriefing were requested.

\section{Discussion}

Emergency and critical care physicians are experiencing increased anxiety related to the pandemic, with disagreement regarding MRP inclusion in the triage team. Our survey was conducted while newscasts and social media publicized disasters in China, Italy, and the United States where patients have died in overwhelmed health systems. ${ }^{2,11-13}$ Most respondents are worried about moral distress if required to ration scarce resources. Fears related to viral contagiousness, decreased family safety, feeling isolated, and maintaining expertise under rapidly changing circumstances were also reported. ${ }^{12,14}$ Canadian 
frontline physicians are seeking clear and consistent guidelines on how to triage scarce critical care resources in COVID-19.

Consistent with recommendations in Canada and the United States, respondents supported triage protocols and multidisciplinary "triage teams" compared to a single "triage officer" and valued the availability of a bioethicist. ${ }^{1,2,15}$ Critical care physicians favored excluding the MRP from these teams, whereas emergency physicians preferred to maintain involvement in triage decisions. Maintaining MRP involvement may be valued in the emergency department due to numerous reasons including differing triage priorities, clinician perspectives, and knowledge gaps. ${ }^{16,17}$ Future qualitative study is needed to understand these divergent preferences in triage team composition, taking into account the context in which these decisions are made.

Greater physician confidence in resource triage decision-making was associated with the availability of triage guidelines. Currently, several consensus-based triage guidelines have been published regionally. ${ }^{1,15}$ However, these guidelines challenge core ethical principles of physician practice such as beneficence and nonmaleficence, with the psychological impact of these practice changes resulting in potentially severe sequalae. ${ }^{2,16}$ Regardless, physicians across both disciplines identified the need for easy to employ, clear and consistent triage guidance, preferentially supported by national regulatory and government authorities.

Our survey found a high rate of anxiety related to the pandemic and the potential rationing of critical care resources, putting physicians at risk for subsequent moral injury. To mitigate existing and anticipated pandemic-related psychological distress amongst physicians; governments, hospitals, and professional associations should collaborate to develop resources such as peer to peer training, professional counselling, and formal debriefing. Literature from first responders and nurses affected by vicarious trauma and post-traumatic stress have supported access to "psychological first aid" training, trauma psychologists, critical incident debriefing, and peer support groups. ${ }^{4,5,12}$ Urgent interdisciplinary collaboration is required to enhance existing triage policies and communicate changes to front line physicians in preparation for a future surge of patients with COVID-19.

This study has several limitations. Non probabilistic sampling inherently leads to sampling error and selection bias, limiting the generalizability of our findings. Duplicate entries may have been included. These limitations were accepted to rapidly activate a short survey to answer pressing questions.

\section{Conclusions}

Canadian physicians responding to COVID-19 are experiencing increased anxiety and are at risk of moral injury. There is a lack of consensus around what role frontline physicians should play in critical care resource allocation decisions. Current guidance on critical care resource triage requires further development to adequately address concerns by prospective users. Urgent collaboration is needed to tailor existing triage guidelines to diverse clinical environments and effectively implement them. 


\section{Declarations}

\section{Conflict of Interest}

The authors have no conflict of interest \& have received no financial support for the study.

\section{Implication Statement}

Physicians engaged in the rationing of scarce critical care resources are expected to be at an increased risk of moral injury. To understand clinician attitudes towards critical care resource triage in COVID-19 and identify areas for subsequent intervention, a national survey of Canadian emergency and critical care physicians was completed.

\section{Acknowledgements}

The primary authorship team would like to thank Mat Mercuri, PhD, McMaster University for methodological review and statistical support.

\section{References}

1. Ontario Health. Clinical Triage Protocol for Major Surge in COVID Pandemic. 2020.

2. Maves RC, Downar J, Dichter JR, Hick JL, Devereaux A, Geiling JA, et al. Triage of Scarce Critical Care Resources in COVID-19 An Implementation Guide for Regional Allocation: An Expert Panel Report of the Task Force for Mass Critical Care and the American College of Chest Physicians. Chest. 2020 Apr 11;(April):1-14.

3. Opatrny L. Triage pour l'accès aux soins intensifs (adultes et pédiatriques) et l'allocation des ressources telles que les respirateurs en situation extrême de pandémie. 2020.

4. Williams RD, Brundage JA, Williams EB. Moral Injury in Times of COVID-19. J Heal Serv Psychol. 2020;1-5.

5. Walton M, Murray E, Christian MD. Mental health care for medical staff and affiliated healthcare workers during the COVID-19 pandemic. Eur Hear J Acute Cardiovasc Care. 2020;9(3):241-7.

6. Valerio MA, Rodriguez N, Winkler P, Lopez J, Dennison M, Liang Y, et al. Comparing two sampling methods to engage hard-to-reach communities in research priority setting. BMC Med Res Methodol. 2016;16(1):1-11.

7. Jager J, Putnick DL, Bornstein MH. II. More Than Just Convenient: the Scientific Merits of Homogeneous Convenience Samples. Monogr Soc Res Child Dev. 2017;82(2):13-30.

8. Harris PA, Taylor R, Minor BL, Elliott V, Fernandez M, O'Neal L, et al. The REDCap consortium: Building an international community of software platform partners. J Biomed Inform. 2019;95:1-10. 
9. Harris PA, Taylor R, Thielke R, Payne J, Gonzalez N, Conde JG. Research electronic data capture (REDCap)-A metadata-driven methodology and workflow process for providing translational research informatics support. J Biomed Inform. 2009;42(2):377-81.

10. Canadian Institute for Health Information. Core Plan: Acute Care Hospitals [Internet]. 2020 [cited 2020 Apr 4]. Available from: https://www.cihi.ca/en/faq/accessing-cihis-products-and-services

11. The Lancet. COVID-19: protecting health-care workers. Lancet. 2020 Mar 21;395(10228):922.

12. Chen Q, Liang M, Li Y, Guo J, Fei D, Wang L, et al. Mental health care for medical staff in China during the COVID-19 outbreak. The Lancet Psychiatry. 2020;7(4):e15-6.

13. Truog RD, Mitchell C, Daley GQ. The Toughest Triage - Allocating Ventilators in a Pandemic. N Engl J Med. 2020 May 21;382(21):1973-5.

14. Shanafelt T, Ripp J, Trockel M. Understanding and Addressing Sources of Anxiety Among Health Care Professionals During the COVID-19 Pandemic. JAMA. 2020 Jun 2;323(21):2133.

15. Sprung CL, Joynt GM, Christian MD, Truog RD, Rello J, Nates JL. Adult ICU Triage During the Coronavirus Disease 2019 Pandemic: Who Will Live and Who Will Die? Recommendations to Improve Survival. Crit Care Med. 2020 May 6;1-7.

16. Frolic A, Kata A, Kraus P. Development of a critical care triage protocol for pandemic influenza: integrating ethics, evidence and effectiveness. Healthc Q. 2009;12(4):54-62.

17. Christian MD. Triage. Crit Care Clin. 2019;35(4):575-89.

\section{Supplementary Files}

This is a list of supplementary files associated with this preprint. Click to download.

- CritResourceTriageCOVIDManuscript20200711SUP.docx 